\title{
Técnica para redução do tônus e alongamento muscular passivo: efeitos na amplitude de movimento de crianças com paralisia cerebral espástica
}

\author{
Technique to reduce tonus and passive muscle stretching: \\ effects on range of motion in children with spastic cerebral palsy \\ Luana dos Santos de Oliveira ${ }^{1}$, Marina Ortega Golin ${ }^{1}$ \\ ${ }^{1}$ Curso de Fisioterapia, Faculdade de Medicina do ABC (FMABC) - Santo André (SP), Brasil.
}

DOI: http://dx.doi.org/10.7322/abcshs.v42i1.946

\begin{abstract}
RESUMO
Introdução: A paralisia cerebral (PC) é resultante de lesão no encéfalo em fase de maturação, acarretando em disfunções motoras. A espasticidade, forma mais comum de acometimento, gera prejuízos funcionais intensificados pela diminuição da mobilidade. Objetivo: Analisar os efeitos do alongamento passivo lento do músculo tríceps sural e de técnica para diminuir o tônus do conceito Bobath na amplitude de movimento (ADM) de dorsiflexão do tornozelo de crianças com PC espástica. Métodos: Participaram 18 crianças atendidas no Hospital Estadual Mario Covas de Santo André e na Santa Casa de Diadema. O grau da hipertonia do músculo tríceps sural foi determinado pela Escala de Aswhorth Modificada e a ADM de dorsiflexão foi medida pela goniometria. Esses dois procedimentos foram realizados antes e após as seguintes situações: 1) aplicação de um protocolo de alongamento muscular passivo; 2) protocolo com uma técnica para diminuir o tônus do conceito Bobath; e 3) emprego associado dos dois protocolos. Resultados: $O$ grau de hipertonia não se modificou após o protocolo 1, porém os protocolos 2 e 3 diminuíram a espasticidade de maneira semelhante, conforme a Escala de Ashworth Modificada. Já o ângulo de dorsiflexão aumentou após aplicação dos três protocolos: $1(p=0,176) ; 2$ ( $p=0,008)$; e 3 , com o aumento mais significativo $(p=0,003)$. Conclusão: A técnica para redução do tônus mostrou efeito positivo na redução da espasticidade, segundo a Escala de Ashworth Modificada, e no aumento da ADM de crianças espásticas. A execução subsequente do alongamento muscular aumentou sua efetividade.
\end{abstract}

Palavras-chave: paralisia cerebral; hipertonia muscular; fisioterapia.

\begin{abstract}
Introduction: Cerebral palsy (CP) is consequent of brain injury in the maturation phase, causing motor dysfunctions. The decreased mobility is intensified by spasticity, most common form of attack, generating functional impairment. Objective: To analyze the effects of slow passive stretching of triceps sure muscle and technique for decreasing the tonus of Bobath concept on the range of motion (ROM) of ankle dorsiflexion in children with spastic CP. Methods: 18 children seen at Hospital Estadual Mario Covas, in Santo André, and Santa Casa, in Diadema, participated in this study. The hypertonia rate of triceps sure muscle was determined by Modified Ashworth Scale and the ROM dorsiflexion by goniometer. Both procedures were realized before and after the following situations: 1) application of a passive muscle stretching protocol; 2) protocol with technique for decreasing the tonus of Bobath concept; and 3) use associated to two protocols. Results: The hypertonia rate did not modify after protocol 1; however, protocols 2 and 3 decreased the spasticity similarly, according to Modified Ashworth Scale. The dorsiflexion angle increased after all protocols: protocol $1(p=0.176)$; protocol $2(p=0.008)$; and protocol 3 , with the most significant increase of ROM ( $p=0.003)$. Conclusion: The technique to reduce tonus shows positive effects on decreasing in hypertonia rate and increase of ROM in spastic children. The subsequent execution of muscle stretching increased its effectiveness.
\end{abstract}

Keywords: cerebral palsy; muscle hypertonia; physical therapy specialty.

Recebido em: 21/03/2016

Revisado em: 01/07/2016

Aprovado em: 21/09/2016

Autor para correspondência: Luana dos Santos de Oliveira - Faculdade de Medicina do ABC - Avenida Lauro Gomes, 2000 - CEP $09060-870$ Santo André (SP), Brasil - E-mail: Iuanafisio93@gmail.com

Conflito de interesses: nada a declarar. 


\section{INTRODUÇÃO}

A paralisia cerebral (PC) é um conjunto de afecções que podem comprometer o encéfalo em fase de maturação estrutural e funcional, devido a uma lesão nos períodos pré, peri ou pós-natal, até o segundo ano de vida. Acarreta disfunções motoras como distúrbios de movimento, postura e tônus. Também podem ocorrer desordens associadas, tais como epilepsias e déficits sensitivos, cognitivos e visuais ${ }^{1,2}$.

Nos países desenvolvidos, cerca de 3 a cada 1.000 nascidos vivos desenvolvem PC. Já em países subdesenvolvidos, devido a um menor acesso aos serviços de saúde, esse índice é ainda mais expressivo: a cada 1.000 nascidos vivos, aproximadamente 7 são acometidos ${ }^{3}$.

Por ser um termo descritivo amplo, a classificação da PC é extremamente variável em relação às manifestações clínicas, à severidade, aos aspectos etiológicos e aos prognósticos, o que torna sua classificação difícil, embora seja imprescindível para complementar o entendimento da mensuração da função motora grossa ${ }^{4}$.

Para descrever sua severidade, modelos tradicionais identificavam a PC como leve, moderada e grave, termos muito subjetivos, utilizados sem consenso entre os profissionais, e que não forneciam informações consistentes sobre quais limitações motoras o paciente apresentava. Por isso, em 1997, um grupo de pesquisadores do Canadá desenvolveu o Sistema de Classificação da Função Motora Grossa (GMFCS), uma escala que classifica a criança em cinco níveis, dependendo da sua idade e das atividades motoras que consegue realizar de forma espontânea e habitual. A diferença entre os cinco níveis baseia-se na capacidade funcional, especialmente no que se refere à postura sentada, à deambulação e à necessidade de dispositivos auxiliares ${ }^{4}$.

Além disso, podemos classificar a PC pela topografia e pelo tipo clínico da lesão. A topografia está relacionada às partes do corpo afetadas, podendo ser: tetraparesia (quatro membros de maneira simétrica), diparesia (quatro membros, predomínio dos inferiores) ou hemiparesia (um hemicorpo) . $^{5}$

Quanto ao tipo clínico, a classificação é baseada no tônus muscular, podendo ser: espástica (lesão no córtex motor, gerando hipertonia elástica), extrapiramidal ou discinética (lesão nos núcleos da base, caracterizada por movimentos involuntários e tônus flutuante), atáxica (lesão no cerebelo ou em suas vias, gerando alterações de equilíbrio, incoordenação e hipotonia), mista (combinação de sinais referentes a diferentes áreas motoras comprometidas) e hipotônica (forma rara, com hipotonia e pobre movimentação) ${ }^{5}$.

Entre as formas apresentadas, a espástica é a mais comum, ocorrendo em cerca de 70\% dos casos. Nessa condição, o paciente apresenta sinais que refletem lesão do motoneurônio superior ${ }^{5}$.

A espasticidade é caracterizada pela tríade: hipertonia elástica (aumento da resistência do músculo ao estiramento passivo), hiper-reflexia (exacerbação dos reflexos miotáticos) e paresia (diminuição da movimentação voluntária). Essa alteração também pode provocar clônus (contrações musculares rítmicas e involuntárias diante do estiramento brusco) e sinal de Babinski (reflexo em leque dos artelhos), fatores que prejudicam o adequado funcionamento muscular ${ }^{5,6}$

Os músculos espásticos de crianças com PC são fracos e apresentam um menor volume muscular, devido a uma combinação entre drive neuronal reduzido, diminuição do tamanho muscular e tensão específica. Medidas diretas revelaram que o comprimento do sarcômero intraoperatório de crianças com PC é mais longo. Verificou-se que, com o crescimento ósseo, em vez de se adicionar sarcômeros em série, é como se eles sofressem alongamento e ocorresse uma desorganização da Matriz Extracelular (MEC), sendo necessário o uso de força muito maior para provocar a contração muscular. Além disso, com o tempo, o aumento da rigidez passiva presente nesses pacientes leva ao surgimento de contraturas e deformidades, causando prejuízos funcionais ${ }^{7,8}$.

Na prática clínica, o tratamento de eleição para minimizar os prejuízos funcionais da espasticidade é o fisioterapêutico. Seu objetivo geral é inibir a atividade reflexa e o tônus anormal, além de estimular o desenvolvimento neuropsicomotor ${ }^{9}$.

Entre as modalidades fisioterapêuticas, a cinesioterapia é adotada com frequência na reabilitação. $\mathrm{O}$ alongamento muscular passivo é uma das principais técnicas utilizadas, sendo de extrema importância para a redução da hipertonia. Além de contribuir para a diminuição da dor, propicia maior mobilidade articular, prevenindo a instalação de contraturas musculares e deformidades ${ }^{9,10}$.

Historicamente, o conceito Bobath é a abordagem cinesioterapêutica mais aplicada em crianças com PC. Criado na década de 1950 por Berta e Karel Bobath, é denominado por inúmeros autores como Tratamento Neuroevolutivo, por ser considerado uma filosofia. Tem como base três pilares conceituais: evitar sinergias musculares atípicas, diminuir a interferência do tônus anormal e facilitar a execução de atividades funcionais ${ }^{11}$.

As técnicas que visam à diminuição da interferência do tônus anormal permitem que a criança se mova de maneira funcional, conseguindo, assim, realizar habilidades motoras específicas. Para diminuir a hipertonia em pacientes espásticos, são empregadas, basicamente, mobilizações passivas lentas e dissociação de cinturas $^{11,12}$.

Apesar de os fisioterapeutas evidenciarem a eficácia dessas técnicas na redução da hipertonia e, consequentemente, no aumento da mobilidade articular, são escassos os registros científicos desses benefícios. Para quantificar seus efeitos, torna-se necessária a adoção de instrumentos de avaliação que mensurem a severidade da hipertonia e a graduação da amplitude de movimento (ADM).

Devido à sua confiabilidade, a Escala de Ashworth Modificada é um dos métodos de avaliação quantitativa da espasticidade mais utilizados na prática clínica. Durante a aplicação dessa escala, é realizada a movimentação passiva rápida da extremidade em análise para observar a $\mathrm{ADM}$ em que a resistência à movimentação aumenta. É subdividida em seis graus de severidade progressiva: $0,1,1+, 2,3$ e $4^{13}$.

Já a goniometria, com o auxílio de um goniômetro, é o método de avaliação mais adotado para a mensuração da ADM. Esse 
instrumento pode quantificar em graus a limitação articular causada pela espasticidade ${ }^{14}$

O emprego associado desses dois instrumentos de avaliação possibilita a delimitação do grau de hipertonia e sua implicação na $\mathrm{ADM}$ do paciente. Vale ressaltar que quanto maior a hipertonia, maior a limitação articular e, consequentemente, maior o prejuízo funcional.

Dessa forma, torna-se claro que a criança espástica apresenta deficiência do controle motor pela diminuição da mobilidade. Assim, é de extrema importância a aplicação de técnicas efetivas para a redução da hipertonia. Esse fato coloca em evidência a necessidade de estudos que quantifiquem os efeitos das diferentes possibilidades de tratamento, para nortear a escolha de propostas que tragam mais benefícios às crianças acometidas. Evidencia-se, então, a relevância de investigar, no presente estudo, os efeitos do alongamento muscular passivo e de técnica de adequação tônica em crianças com PC espástica.

O objetivo deste estudo foi analisar, em crianças com PC espástica, os efeitos do alongamento muscular passivo lento do músculo tríceps sural e de técnica para diminuir o tônus do conceito Bobath na ADM de dorsiflexão de tornozelo.

\section{MÉTODOS}

Trata-se de um estudo transversal observacional e controlado. Participaram da pesquisa 18 crianças com diagnóstico de PC espástica atendidas no Hospital Estadual Mário Covas de Santo André e na Santa Casa de Misericórdia de Diadema, ambos localizados no estado de São Paulo.

Para a seleção, os critérios de inclusão adotados foram: realizar tratamento fisioterapêutico nos ambulatórios citados, diagnóstico médico de PC, diagnóstico clínico de espasticidade, assinatura prévia do Termo de Consentimento Livre e Esclarecido pelo responsável e, em casos de crianças entre 7 e 17 anos, assinatura também do Termo de Assentimento. Já os de exclusão foram: não colaboração da criança na execução das técnicas, presença de deformidade articular no tornozelo, retração tecidual dos músculos flexores plantares que não permitisse a dorsiflexão passiva a neutro ou zero grau após diminuição da hipertonia, retração tecidual dos músculos flexores do joelho que impossibilitasse a extensão passiva total do joelho com o quadril em posição neutra e ter sido submetido à aplicação de toxina botulínica no músculo tríceps sural no período de seis meses.

Os procedimentos foram aprovados pelo Comitê de Ética em Pesquisa da Faculdade de Medicina do ABC (FMABC), sob o protocolo $\mathrm{n}^{\circ}$ 42987214.9.0000.0082.

Todos os participantes foram classificados de acordo com os níveis funcionais do GMFCS e submetidos a três protocolos de intervenções fisioterapêuticas, realizados em dias diferentes. Antes e após a aplicação de cada protocolo foram mensurados o grau de hipertonia do músculo tríceps sural dos participantes, via Escala de Aswhorth Modificada, e o ângulo de dorsiflexão passiva, via goniometria convencional realizada de forma adaptada, visto que na maior parte da amostra não foi possível colocar o tornozelo em posição neutra antes da aplicação das técnicas, o que resultaria em valores de dorsiflexão negativos. Desse modo, os participantes foram posicionados em decúbito dorsal, com o joelho direito em extensão e com o tornozelo em plantiflexão máxima; o goniômetro foi posicionado com o braço fixo em alinhamento com a tíbia e o braço móvel em alinhamento com o quinto metatarso, sendo a medida realizada partindo da plantiflexão para a máxima dorsiflexão. Portanto, sabendo que o ângulo de dorsiflexão é de $20^{\circ}$ quando o tornozelo é posicionado em neutro $\left(90^{\circ}\right)$, o ângulo de $80^{\circ}$, por exemplo, descrito neste trabalho, seria equivalente $\mathrm{a}-10^{\circ}$ de dorsiflexão $\mathrm{o}^{15-17}$.

No protocolo 1 foi realizado o alongamento muscular passivo lento do tríceps sural, durante 30 segundos, totalizando 5 repetições (Figura 1). No protocolo 2 foi aplicada uma técnica para diminuir o tônus do conceito Bobath, em que as crianças foram posicionadas em decúbito lateral, com um dos membros inferiores (MMII) apoiado em alinhamento no tablado e outro em abdução, semiflexão e rotação externa de quadril, joelho flexionado e pé apoiado, com transferência de peso e aumento gradual da ADM (Figura 2); o tempo de execução da técnica foi de 5 minutos. No protocolo 3 foi realizada a aplicação da mesma técnica de adequação tônica, seguida pelo protocolo de alongamento muscular, ou seja, o emprego associado dos dois protocolos. As técnicas foram realizadas no membro inferior direito (MID) e no membro inferior esquerdo (MIE), porém, para evitar o descarte da amostra de hemiparéticos, foi padronizado apenas o MID.

Para análise dos resultados, as variáveis qualitativas foram apresentadas por frequência absoluta e relativa, e as quantitativas, por mediana e intervalo de confiança de 95\% (IC95\%), percentis 25 e 75, por não apresentarem distribuição normal (Shapiro-Wilk;

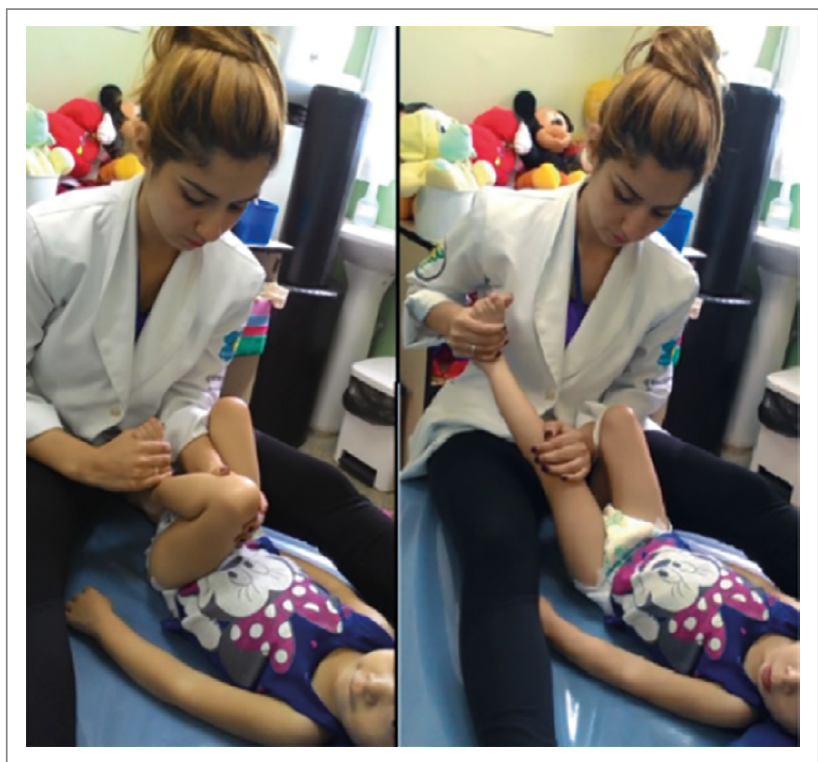

Figura 1: Posições inicial e final da técnica de alongamento muscular passivo lento do músculo tríceps sural 
$\mathrm{p}<0,05)$. A associação entre as variáveis qualitativas foi avaliada pelo teste do $\chi^{2}$. Para comparar a angulação inicial e após a aplicação de cada protocolo, utilizou-se o teste de Wilcoxon. As diferenças de angulação (resultantes da angulação final menos a angulação inicial) entre os protocolos foram comparadas pelo teste de Kruskal-Wallis. A análise estatística foi realizada no software estatístico Stata, versão 11.0.

\section{RESULTADOS}

Dentre os 18 participantes, a mediana de idade foi de 3 anos e 10 crianças eram do sexo feminino (55,6\%). Quanto à topografia da lesão, ou seja, os seguimentos corporais acometidos pela função motora, 8 participantes eram tetraparéticos $(44,4 \%)$, 6, diparéticos $(33,3 \%)$, e 4, hemiparéticos (22,3\%). Em relação aos níveis funcionais do GMFCS, 3 crianças foram classificadas como nível I, 2 como II, 3 como III, 2 como IV e 8 como V.

A Tabela 1 mostra a frequência dos graus de espasticidade, avaliados antes e após a realização dos três protocolos, segundo a Escala de Ashworth Modificada. Nenhum participante apresentou

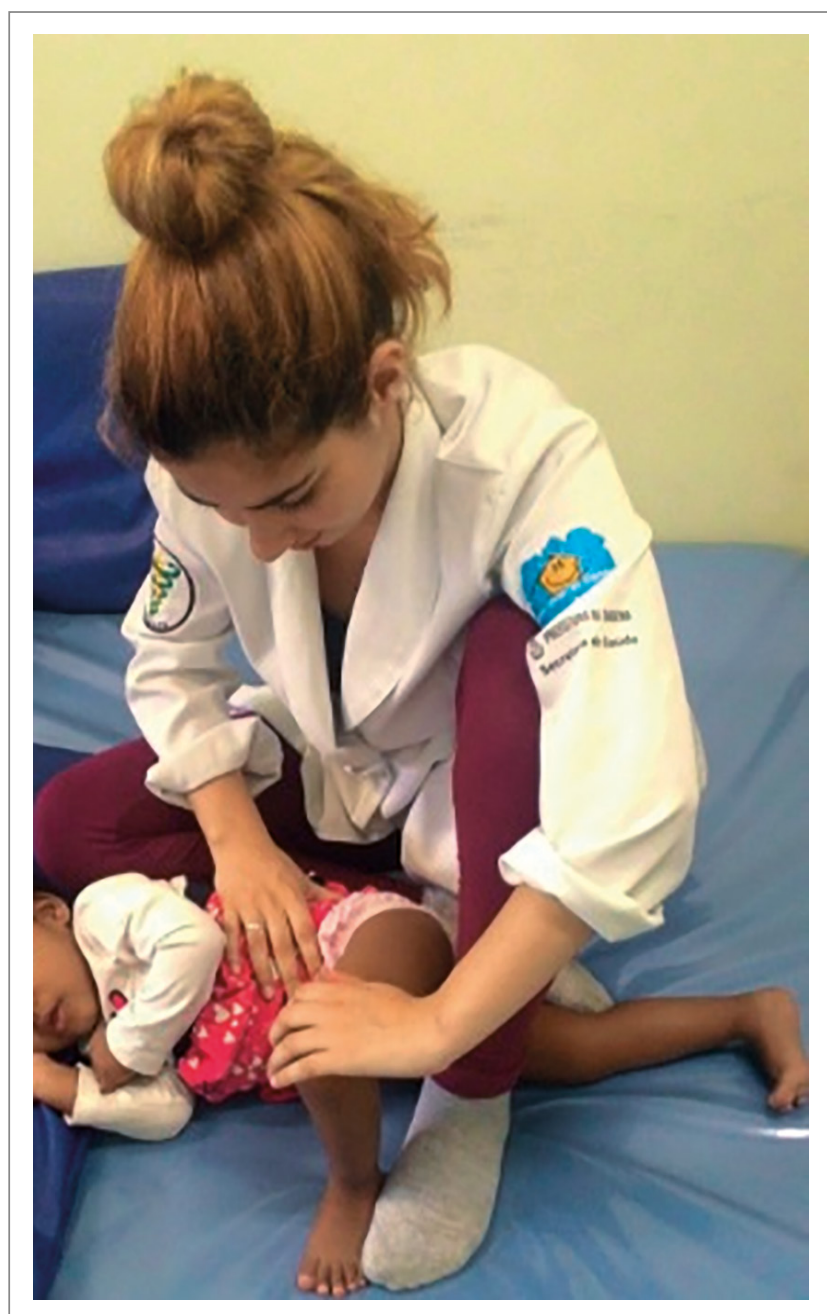

Figura 2: Técnica para diminuir o tônus, segundo o conceito Bobath redução do grau de hipertonia após a aplicação do protocolo de alongamento muscular. No entanto, os protocolos de adequação tônica e de adequação tônica+alongamento muscular foram capazes de alterar positivamente e de maneira semelhante $(p<0,001)$ o grau de espasticidade dos participantes.

Na Tabela 2 é possível evidenciar a evolução na espasticidade dos participantes após a aplicação das três técnicas, ou seja, se conseguiram diminuir a graduação da hipertonia. Comparando as três técnicas fisioterapêuticas, houve diferença estatisticamente significante, mas a técnica de alongamento muscular não foi capaz de alterar o grau de espasticidade. Já a técnica para diminuir o tônus e o emprego associado das duas técnicas foi capaz de diminuir a espasticidade de 14 participantes.

$\mathrm{Na}$ Tabela 3 são mostradas a mediana e as estimativas pontuais e intervalares dos valores correspondentes ao ângulo de dorsiflexão do tornozelo direito dos participantes da pesquisa, antes e após a aplicação das técnicas. Após a aplicação do alongamento muscular, a mediana do ângulo de dorsiflexão aumentou, porém sem significância estatística $(\mathrm{p}=0,176)$. A técnica para diminuir o tônus apresentou maior efetividade e foi significante no aumento da angulação quando comparada ao alongamento muscular $(\mathrm{p}=0,008)$. Já a associação da aplicação da técnica para diminuir o tônus+alongamento muscular mostrou-se ainda mais efetiva, com nível de significância maior $(\mathrm{p}=0,003)$.

Tabela 1: Frequência absoluta e relativa da Escala de Ashworth Modificada antes e após a aplicação dos protocolos

\begin{tabular}{|c|c|c|c|c|c|c|}
\hline \multirow{3}{*}{$\begin{array}{l}\text { Escala } \\
\text { Ashworth } \\
\text { Modificada* }\end{array}$} & \multicolumn{2}{|c|}{$\begin{array}{l}\text { Alongamento } \\
\text { muscular }\end{array}$} & \multicolumn{2}{|c|}{$\begin{array}{l}\text { Redução } \\
\text { do tônus }\end{array}$} & \multicolumn{2}{|c|}{$\begin{array}{l}\text { Redução do tônus+ } \\
\text { alongamento } \\
\text { muscular }\end{array}$} \\
\hline & Antes & Após & Antes & Após & Antes & Após \\
\hline & \multicolumn{6}{|c|}{ n (\%) } \\
\hline 1 & $\begin{array}{c}04 \\
(22,2)\end{array}$ & $\begin{array}{c}04 \\
(22,2)\end{array}$ & $\begin{array}{c}04 \\
(22,2)\end{array}$ & $\begin{array}{c}17 \\
(94,4)\end{array}$ & $\begin{array}{c}04 \\
(22,2)\end{array}$ & $\begin{array}{c}17 \\
(94,4)\end{array}$ \\
\hline $1+$ & $\begin{array}{c}13 \\
(72,2)\end{array}$ & $\begin{array}{c}13 \\
(72,2)\end{array}$ & $\begin{array}{c}13 \\
(72,2)\end{array}$ & $\begin{array}{c}01 \\
(5,6)\end{array}$ & $\begin{array}{c}13 \\
(72,2)\end{array}$ & $\begin{array}{c}01 \\
(5,6)\end{array}$ \\
\hline 2 & $\begin{array}{c}01 \\
(5,6)\end{array}$ & $\begin{array}{c}01 \\
(5,6)\end{array}$ & $\begin{array}{c}01 \\
(5,6)\end{array}$ & $0(0)$ & $\begin{array}{c}01 \\
(5,6)\end{array}$ & $\begin{array}{c}0 \\
(0)\end{array}$ \\
\hline Valor $p^{\star \star}$ & \multicolumn{2}{|c|}{$<0,001$} & \multicolumn{2}{|c|}{$<0,001$} & \multicolumn{2}{|c|}{$<0,001$} \\
\hline
\end{tabular}

*Os níveis 3 e 4 da Escala de Ashworth Modificada foram desconsiderados na análise, em virtude de nenhum paciente apresentar essas categorias. ${ }^{\star *}$ Valores probabilísticos do teste do $\chi^{2}$

Tabela 2: Associação entre as técnicas fisioterapêuticas realizadas e a diminuição da espasticidade dos participantes

\begin{tabular}{|l|c|c|}
\hline \multirow{2}{*}{ Técnicas fisioterapêuticas } & \multicolumn{2}{|c|}{ Diminuição* } \\
\hline $\begin{array}{l}\text { Não } \\
\mathbf{n}(\%)\end{array}$ & $\begin{array}{c}\text { Sim } \\
\mathbf{n}(\%)\end{array}$ \\
\hline $\begin{array}{l}\text { Rlongamento muscular } \\
\text { Redução do tônus }\end{array}$ & $18(100)$ & $0(0)$ \\
\hline $\begin{array}{l}\text { Redução do tônus+ } \\
\text { alongamento muscular }\end{array}$ & $4(22,2)$ & $14(77,8)$ \\
\hline $\mathrm{p}^{\text {** }}$ & $4(22,2)$ & $14(77,8)$ \\
\hline
\end{tabular}

*Os pacientes que não apresentaram diminuição mantiveram o grau de espasticidade, classificado segundo a Escala de Ashworth Modificada; ${ }^{* *}$ valores probabilísticos do teste do $\chi^{2}$. 
A Figura 3 mostra que as três técnicas foram capazes de aumentar o ângulo de dorsiflexão do pé direito dos participantes; porém, após a aplicação da técnica para diminuir o tônus+alongamento muscular, o incremento da angulação teve maior significância.

\section{DISCUSSÃO}

Os resultados do presente estudo sugerem que a técnica de alongamento muscular passivo lento aplicado no músculo tríceps sural é eficaz na diminuição da espasticidade, pois promoveu aumento da ADM do tornozelo dos participantes. A técnica para diminuir o tônus do conceito Bobath aplicada mostrou-se eficaz para o tratamento da espasticidade, uma vez que após sua aplicação foi possível observar, além de aumento do ângulo de dorsiflexão passiva, uma diminuição do grau da espasticidade, segundo a Escala de Ashworth Modificada. No entanto, após a aplicação das duas técnicas associadas, os resultados foram ainda melhores, pois o aumento do ângulo de dorsiflexão foi maior e o grau de espasticidade também diminuiu.

O presente estudo constatou que o alongamento muscular passivo lento do músculo tríceps sural foi capaz de diminuir a espasticidade, pois, mesmo não havendo alteração significativa na Escala

Tabela 3: Estimativa pontual e intervalar da angulação de dorsiflexão do pé direito antes e após a aplicação dos protocolos

\begin{tabular}{|c|c|c|c|}
\hline \multirow{2}{*}{$\begin{array}{l}\text { Técnicas } \\
\text { fisioterapêuticas }\end{array}$} & Antes & Após & \multirow{2}{*}{$\begin{array}{c}\text { Valor } \\
\mathbf{p}^{\star}\end{array}$} \\
\hline & \multicolumn{2}{|c|}{ Mediana (IC95\%) } & \\
\hline $\begin{array}{l}\text { Alongamento } \\
\text { muscular }\end{array}$ & $\begin{array}{c}88,5^{\circ} \\
\left(80,0^{\circ}-90,7^{\circ}\right)\end{array}$ & $\begin{array}{c}92^{\circ} \\
\left(85,9^{\circ}-96,0^{\circ}\right)\end{array}$ & 0,176 \\
\hline Redução do tônus & $\begin{array}{c}87,5^{\circ} \\
\left(80,0^{\circ}-92,0^{\circ}\right)\end{array}$ & $\begin{array}{c}98^{\circ} \\
\left(92,3^{\circ}-102,1^{\circ}\right)\end{array}$ & 0,008 \\
\hline $\begin{array}{l}\text { Redução do } \\
\text { tônus+alongamento } \\
\text { muscular }\end{array}$ & $\begin{array}{c}88^{\circ} \\
\left(81,2^{\circ}-93,4^{\circ}\right)\end{array}$ & $\begin{array}{c}109^{\circ} \\
\left(98,6^{\circ}-119,7^{\circ}\right)\end{array}$ & 0,003 \\
\hline
\end{tabular}

*Valores probabilísticos do teste de Wilcoxon; ${ }^{* *}$ goniometria adaptada feita partindo da plantiflexão para a máxima dorsiflexão;

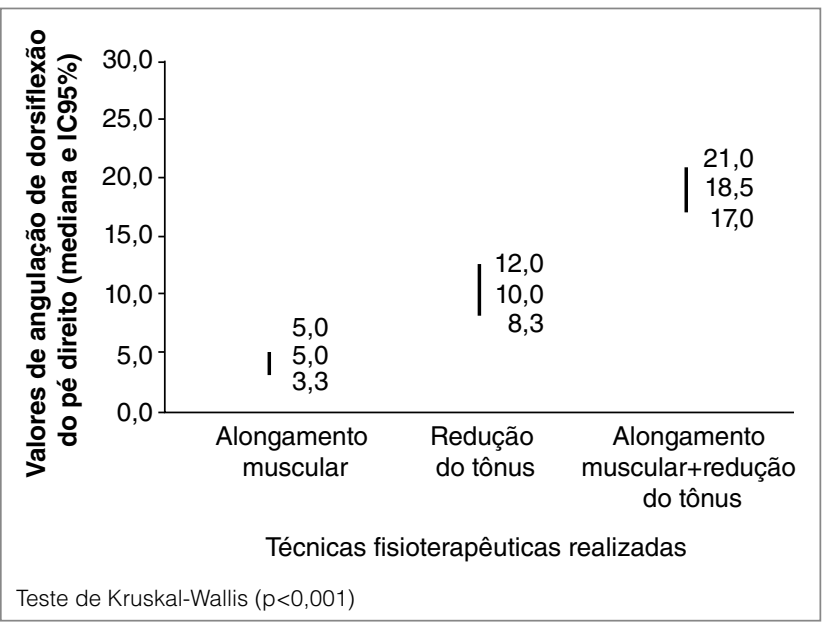

Figura 3: Estimativa pontual e intervalar da angulação de dorsiflexão do pé direito com valores mais significativos após o protocolo alongamento muscular e redução do tônus de Ashworth Modificada, foi possível observar aumento do grau de dorsiflexão passiva dos participantes pela goniometria convencional (uma medida indireta de graduar a espasticidade). Isso porque quanto maior a espasticidade desse músculo, mais intenso será o reflexo do estiramento, com consequente contração muscular e menor angulação de dorsiflexão.

Smania et al. ${ }^{18}$ apontam os métodos de reabilitação mais utilizados no manejo da espasticidade e referem que, nas últimas décadas, vários procedimentos não farmacológicos foram apresentados na literatura, incluindo o alongamento muscular passivo. Essa técnica também gera melhora da flexibilidade muscular, sendo inclusive ótima escolha na prevenção de contraturas e deformidades $\operatorname{articulares}^{18}$.

Vários estudos relatam os benefícios que o alongamento muscular pode gerar. Sèze et al. ${ }^{19}$, por exemplo, afirmam que as técnicas de alongamento muscular propõem manter ou melhorar a extensibilidade músculo-facial e a amplitude de movimento, promovendo melhora nas habilidades funcionais, além de atrasar e/ou evitar intervenções cirúrgicas ${ }^{19-22}$.

Um ponto observado em muitos trabalhos, como no de Segura et $a .^{23}$, é que os resultados mostram que o alongamento muscular gera aumento da flexibilidade muscular e consequente diminuição da hipertonia, porém esses autores não relatam detalhes da realização da técnica, como, por exemplo, o posicionamento realizado, a duração e o número de repetições, o que dificulta uma reprodução exata ${ }^{23}$.

Neste estudo, no entanto, após a aplicação do protocolo de alongamento, apesar ter sido verificado aumento do ângulo de dorsiflexão muscular, não houve redução da hipertonia, segundo a Escala de Ashworth Modificada. Isso pode ter ocorrido, pois, segundo alguns pesquisadores, essa escala não é muito sensível às alterações pequenas de tensão muscular e, por isso, vem se tornando alvo constante de críticas $^{24}$.

Dessa forma, os resultados aqui encontrados sugerem que a goniometria convencional, por ser uma estratégia de avaliação quantitativa, foi capaz de mensurar pequenas reduções na hipertonia. Já a Escala de Ashworth Modificada foi sensível apenas quando os efeitos gerados

pela técnica causaram grandes alterações no tônus muscular, visto que os pacientes que apresentaram diminuição na graduação da escala tiveram significativos aumentos nos graus de amplitude de dorsiflexão.

Um estudo de revisão publicado por Pin et al. ${ }^{25}$ corrobora os resultados encontrados no presente estudo, pois, após analisarem sete publicações com o objetivo de avaliar as evidências sobre a eficácia do alongamento muscular passivo em crianças com PC, os autores concluíram que parece haver algumas evidências de que essa estratégia de tratamento pode aumentar a ADM e reduzir a espasticidade ${ }^{25}$.

O conceito Bobath de tratamento é historicamente o mais aplicado em crianças com PC e sua principal meta é ganhar a maior independência funcional possível. Com base nesse conceito, outra técnica aplicada neste estudo foi a de redução de tônus, uma estratégia 
fundamental para tratamento de disfunções neuromotoras, pois, ao minimizar a interferência da hipertonia, proporciona melhor mobilidade ao paciente, facilitando, assim, ganhos funcionais ${ }^{26,27}$.

Após a aplicação do protocolo com a técnica para redução do tônus do conceito Bobath, foi possível observar uma redução da hipertonia dos participantes, o que corrobora os resultados de Peres et $a^{28}$, que verificaram, segundo a Escala de Ashworth Modificada, os efeitos desse conceito no tônus de quatro crianças diparéticas espásticas portadoras de PC, e também apontaram uma diminuição da hipertonia após a utilização desse conceito.

No entanto, muitos dos estudos que utilizaram o conceito Bobath, como o citado acima, adotam tal conceito para o tratamento de crianças com PC e, afirmam ter encontrado resultados positivos após sua aplicação, porém não especificam qual técnica desse conceito foi utilizada nem como ela foi aplicada ${ }^{28-30}$.

O presente estudo, ao utilizar a técnica para diminuir o tônus de maneira isolada, verificou, por meio da goniometria convencional, aumento estatisticamente significante no ângulo de dorsiflexão dos participantes. Comparando-se os resultados encontrados após a aplicação do alongamento muscular de maneira isolada, o aumento do ângulo foi maior após a técnica de adequação, que também diminuiu o grau de espasticidade observado pela Escala de Ashworth Modificada em mais de 50\% dos participantes.

Após a aplicação do protocolo com a associação das duas técnicas foi possível observar, além da diminuição do grau de espasticidade, pela Escala de Ashworth Modificada, aumento mais significativo da ADM de dorsiflexão dos participantes. Tal resultado sugere que essa técnica é a melhor escolha, pois o aumento imediato da ADM não pode ser associado ao aumento da elasticidade muscular, e sim à diminuição da contração reflexa do músculo causada pela espasticidade.

Além disso, de acordo com os relatos da avaliadora, foi possível observar melhor aceitação das crianças aos manuseios e uma maior facilidade para realizar o alongamento muscular quando a técnica para redução do tônus foi realizada antes do alongamento muscular, provavelmente pelo relaxamento muscular proporcionado por ela.
Em estudo semelhante publicado por Dalesse et al. ${ }^{31}$, a amostra foi composta por 24 crianças com PC espástica, divididas em 2 grupos aleatórios. Um dos grupos foi submetido a um protocolo de alongamento muscular passivo do tríceps sural e o outro a um protocolo de alongamento muscular passivo associado ao emprego de uma técnica para adequação tônica do conceito Bobath. Os resultados foram mensurados apenas pela Escala de Ashworth Modificada e foi observada diminuição da graduação da espasticidade após a aplicação dos dois protocolos, não havendo diferença estatisticamente significante entre os grupos, sugerindo, assim, que a adoção de qualquer uma das técnicas proporciona diminuição da espasticidade. No entanto, a não mensuração da ADM de dorsiflexão pode ter consistindo em viés para análise da real diferença entre os resultados obtidos pelos protocolos ${ }^{31}$.

Contudo, foi possível concluir que a aplicação de técnica para redução do tônus prévia ao alongamento muscular passivo lento do tríceps sural, segundo o conceito Bobath, aumenta a efetividade da redução da espasticidade desse músculo, promovendo, consequentemente, melhora da mobilidade articular. Verifica-se, portanto, que a aplicação das duas técnicas de maneira associada, como foi realizado no protocolo 3, promove resultados melhores.

Uma das limitações metodológicas do presente estudo foi a dificuldade em se obter uma amostra mais homogênea quanto à severidade do comprometimento motor, isso devido ao fluxo de atendimento das duas instituições nas quais a pesquisa foi realizada. Para maior controle das variáveis nos resultados, o ideal seria avaliar pacientes pertencentes ao mesmo nível funcional do GMFCS.

Além disso, os dados na literatura quanto aos reais efeitos das técnicas fisioterapêuticas manuais na redução da hipertonia ainda são escassos. Por isso, é extremamente importante realizar novos estudos nesse âmbito, para a real comprovação dessas técnicas, bem como utilizar outros métodos de avaliação como, por exemplo, o flexímetro para mensuração da angulação articular, por ser um instrumento mais fidedigno em relação à goniometria passiva. Dessa forma, será possível que o profissional se sinta seguro na adoção de técnicas que realmente tragam efeitos benéficos aos seus pacientes.

\section{REFERÊNCIAS}

1. Fonseca JO, Cordani LK, Oliveira MC. Aplicação de um inventário de avaliação pediátrica de incapacidade (PEDI) com crianças portadores de paralisia cerebral espástica. Rev Ter Ocup Univ. 2005;16(2):67-74. http://dx.doi.org/10.11606/issn.2238-6149.v16i2p67-74

2. Leite JMRS, Prado GF. Paralisia cerebral aspectos fisioterapêuticos e clínicos. Rev Neurocienc. 2004;12(1):41-5 http://dx.doi.org/10.4181/RNC.2004.12.41

3. Assis-Madeira EA, Carvalho SG. Paralisia cerebral e fatores de riscos ao desenvolvimento motor: uma revisão teórica. Cad PósGraduação Distúrbios Desenv. 2009;9(1):142-63.

4. Monteiro CBM, Abreu LCA, Valenti VE. Paralisa cerebral: teoria e prática. Plêiade; 2015. p.115-6
5. Gomes CO, Golin MO. Tratamento fisioterapêutico na paralisia cerebral tetraparesia espástica, segundo conceito Bobath. Rev Neurocienc. 2013;21(2):278-85

http://dx.doi.org/10.4181/RNC.2013.21.757.8p

6. Gois DNA, Cândido EAF, Cândido JF, Oliveira TVC. Analise do tratamento fisioterapêutico em crianças com paralisia cerebral do tipo hemiparética espásticas. Scire Salutis. 2011;1(2):15-22. http://dx.doi.org/10.6008\%2FESS2236-9600.2011.002.0002

7. Smith LR, Lee KS, Ward SR, Chambers HG, Lieber RL. Hamstring contractures in children with sapastic cerebral palsy result from a stiffer extracelular matrix and increased in vivo sarcomere length. $J$ Physiol. 2011;589(10):2625-39.

http://dx.doi.org/10.1113/jphysiol.2010.203364 
8. Lucas RS, Eva P, Yvett H, Samuel RW, Henry GC, Shankar S, et al. Novel transcriptional profile in wrist muscles from cerebral palsy patients. BMC Med Genomics. 2009;2:44. http://dx.doi.org/10.1186/1755-8794-2-44

9. Franco CB, Pires LC, Pontes LS, Sousa EJ. Avaliação da amplitude articular do tornozelo em crianças com paralisia cerebral após a aplicação de toxina botulínica seguida de fisioterapia. Rev Para Med. 2006;20(3):43-9.

10. Lianza S. Consenso Nacional sobre Espasticidade. Diretrizes para diagnósticos e tratamentos. São Paulo: SBMFR; 2001. p.15-38.

11. Dias AAS, Rodrigues GR. Revisão bibliográfica sobre o Método Bobath: à luz da fisioterapia na encefalopatia crônica da infância tipo diplegia espástica de 0 (zero) a 3 anos. Monografia (Conclusão de Curso) - Universidade Veiga de Almeida, Rio de Janeiro, 2007. p. $32-4$

12. Santos LPD, Golin MO. Evolução motora de crianças com paralisia cerebral diparesia espástica. Rev Neurocienc. 2013;21(2):184-92. http://dx.doi.org/10.4181/RNC.2013.21.808.9p

13. Associação Brasileira de Medicina Física e Reabilitação (ABMFR) Espasticidade: avaliação clínica. Projeto Diretrizes. ABMFR; 2006.

14. Ferrarezi KC, Guedes JERP. O uso de técnicas para auxiliar a flexibilidade e equilíbrio em adolescentes portadores de paralisia cerebral: o relato de três casos. Acta Scientiarum. 2000; 2(22):625-29.

http://dx.doi.org/10.4025/actascibiolsci.v22i0.3095

15. Marques AP. Ângulos articulares dos membros inferiores. In: Marques AP. Manual de goniometria. 2. ed. São Paulo: Manole; 2003. p. 41-7.

16. Magee DJ. Perna, tornozelo e pé. In: Magee DJ, editor. Disfunção musculoesquelética. 3. ed. São Paulo: Manole; 2002. p. 621-95.

17. Palmer LM, Epler ME. Tornozelo e pé. In: Palmer LM, Epler ME. Fundamentos das Técnicas de Avaliação Musculoesquelética. 2. ed. Rio de Janeiro: Guanabara Koogan; 2000. p. 302-36.

18. Smania N, Picelli A, Munari D, Geroin C, lanes P, Waldner A, et al. Rehabilitation procedures in the management of spasticity. Eur $\mathrm{J}$ Phys Rehabil Med. 2010;46(3):423-38.

19. Sèze M, Wiart L, Bon-Saint-Côme A, Debelleix X, de Sèze M, Joseph $\mathrm{PA}$, et al. Rehabilitation of postural disturbances of hemiplegic patients by using trunk control retraining during exploratory exercises. Arch Phys Med Rehabil. 2001;82(6):793-800. http://dx.doi.org/10.1053/apmr.2001.0820793
20. Nunes LE, Martins RAS, Macedo AB. A eficácia da associação das técnicas de alongamento, facilitação neuromuscular proprioceptiva e controle postural em adolescente com hemiparesia: estudo de caso. Rev Saúde CESUC. 2010;1:1-10

21. Falsarella GR, Ramos MG, Páscoa MA, Vecchio FBD. Estudo experimental de grupo único com avaliação pré e pós: através da prática do alongamento na extensão universitária FEF/Unicamp. Conexões. 2008;6(1):79-85. http://dx.doi.org/10.20396/conex.v6i1.8637873

22. Junqueira RT, Ribeiro AMB, Scianni AA. Efeitos do fortalecimento muscular e sua relação com a atividade funcional e a espasticidade em indivíduos hemiparéticos. Rev Bras Fisioter. 2004;8(3):247-52.

23. Segura DCA, Crespão DL, Darolt M, Beledel AS, Piccin AS, Picinini JAS. Análise do tratamento da espasticidade através da fisioterapia e da farmacologia: um estudo de caso. Rev Ciênc Saúde Unipar. 2007;11(3):217-24.

24. Platz T, Eickhof C, Nuyens G, Vuadens P. Clinical scales for the assessment of spasticity, associated phenomena, and function: a systematic review of the literature. Disabil Rehabil. 2005;27(1-2):7-18.

25. Pin $T$, Dyke $P$, Chan $M$. The effectiveness of passive stretching in children with cerebral palsy. Deve Med Child Neurol. 2006;48(10):855-62. http://dx.doi.org/10.1017/S0012162206001836

26. Infurta T, Hacker D,Golineleo MT. Análise do percentual do GMFM (dimensões D E E) em uma criança pc submetida à fisioterapia. Rev Bras Fisioter. 2007;11(suppl.):109.

27. Krigger KW. Cerebral palsy: an overview. Am Fam Physician 2006;73(1):91-100

28. Peres LW, Ruedell AM, Diamante C. Influência do conceito neuroevolutivo Bobath no tônus e força muscular e atividades funcionais estáticas e dinâmicas em pacientes diparéticos espásticos após paralisia cerebral. Saúde. 2009;35(1):28-33.

29. Duarte MP, Rabello LM. Conceito neuroevolutivo Bobath e a facilitação neuromuscular proprioceptiva como forma de tratamento para crianças com encefalopatia crônica não progressiva da infância. Rev Científica FAEMA. 2015;6(1):14-26.

30. Valverde ME, Serrano MP. Terapia de neurodesarrollo: concepto Bobath. Past Rest Neurol. 2003;2(2):139-42.

31. Dalesse ACE, Grecco LAC, Oliveira CS, Golin MO. Efeitos de técnicas fisioterapêuticas para reduzir a hipertonia em crianças com paralisia cerebral. Rev Terapia Manual Posturologia. 2013;11(51):100-4. 